\title{
Urethral catheterization of the male ferret for treatment of urinary tract obstruction
}

\author{
Cyndi Brown, DVM, Dipl. ABVP-Avian ${ }^{1}$ \& Christal Pollock, DVM, Dipl. ABVP-Avian ${ }^{2}$
}

\begin{abstract}
Male ferrets are used in various biomedical research studies and generally thrive in laboratory conditions. Urethral obstruction can occur in male ferrets of all ages, and urethral catheterization may be needed to relieve the obstruction. This column describes urinary catheter selection, placement and monitoring in the male ferret.
\end{abstract}

The domestic ferret (Mustela putorius furo) has been used as a model in biomedical research, including studies of human influenza and severe acute respiratory syndrome-associated corona virus. Ferrets have served as models for peptic ulcer disease, carotenoid metabolism, cystic fibrosis and drug emesis screening, and they make excellent study models for virology and immunology. Ferrets have a distinct niche in biomedical research and are hardy animals that thrive in the laboratory. Most research ferrets are males because females have estrus-related health problems ${ }^{1}$.

Urethral obstruction occurs in male ferrets of all ages, most commonly in those older than 2 years, typically secondary to adrenal disease and androgenic stimulation of the prostatic tissue that surrounds the urethra ${ }^{2}$. Inflammation of this prostatic tissue can lead to compression of the urethra and a functional obstruction of urine outflow. Urinary tract obstruction in the ferret may also be the result of struvite urolithiasis, but the incidence of this disorder is relatively low.

\section{Clinical signs}

Clinical signs associated with urethral obstruction include stranguria, pollakiuria, dysuria, alopecia and pruritis (commonly associated with adrenal disease), urine dribbling, urine scald, frequent licking of the prepuce, hematuria and anuria. Ferrets with complete urethral obstruction may strain violently or cry when attempting to urinate. The straining may be misinterpreted as constipation. Tenesmus may even lead to diarrhea in some cases.

\section{Diagnostic testing}

The most common abnormal finding on physical examination is distension and pain of the urinary bladder. For diagnosis, samples should be submitted for complete blood count, serum biochemistry, urinalysis and urine bacterial culture and sensitivity. A ferret androgen panel can be submitted to the University of Tennessee for confirmation of adrenal gland disease.

Abdominal ultrasound and radiography are valuable diagnostic tools that allow evaluation of the urinary tract, prostate and adrenal glands (as well as the rest of the abdominal viscera). Calculi lodged at the os penis can be difficult to detect. Contrast radiography may be useful in identifying urethral stones.

\section{Urinary catheter preparation}

Urethral catheter placement is challenging in the male ferret due to the animal's small size and J-shaped os penis (Fig. 1). General anesthesia is required to achieve adequate skeletal muscle relaxation for urinary catheter placement. The ferret should be closely monitored while under anesthesia; the electrocardiogram should be monitored for evidence of hyperkalemia (such as loss of the $\mathrm{P}$ wave, widening of the QRS complex).

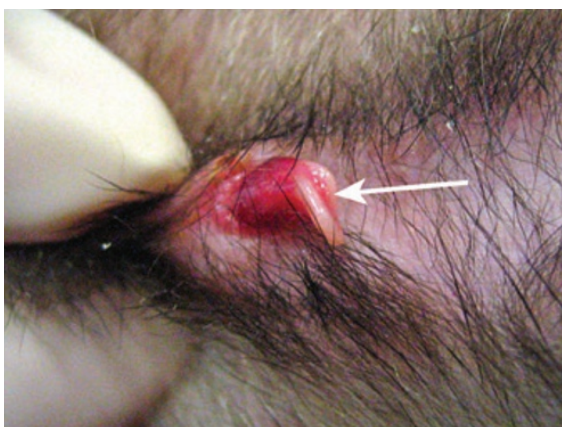

FIGURE 1 | Close-up image (ventral view) of the tip of a ferret's penis (extruded from the prepuce while the ferret was under anesthesia), showing the two projections of the os penis and the groove between them. The urethral opening lies on the ventral aspect just proximal to the tip of the penis within the groove created by the two osseous structures (arrow).

Relief of the urinary tract obstruction and forced diuresis are usually sufficient to manage the hyperkalemia. Medical treatment for hyperkalemia is indicated if an arrhythmia is present in addition to poor perfusion or altered mental state.

\section{Urinary catheter selection}

A urinary catheter designed for use in ferrets, such as the $3.0-\mathrm{Fr}, 11-\mathrm{cm}$, open-ended silicone catheter known as the 'Slippery Sam' (Global Veterinary Products; New Buffalo, MI), should be used whenever possible ${ }^{3}$. The size and flexibility of this catheter make it ideal. Other catheters such as the 3.0-Fr tomcat urethral catheter (which may

${ }^{1}$ Ocean State Veterinary Specialists, East Greenwich, RI. ${ }^{2}$ Veterinary consultant, Cleveland Heights, OH. Correspondence should be addressed to C.P. (christal7@mac.com). 


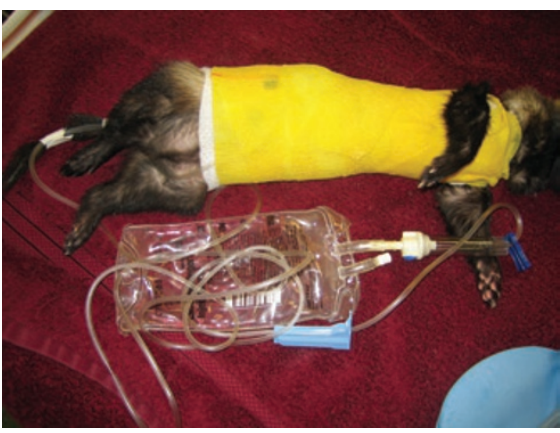

FIGURE 2 | An anesthetized ferret with a body wrap to protect the urinary catheter. The bandage extends from the caudal abdomen to the thorax and up over the shoulders to prevent it from slipping down. There is a loose loop of tubing taped within the ventral aspect of the bandage, and the tubing is also secured to the tail for protection.

not be long enough for all ferrets), a 20 - or 22-gauge, 8-in jugular catheter or a 3.5-Fr red rubber catheter can be successfully used but are more likely to result in perforation of the urethra because they are less flexible. When used as urinary catheters, red rubber and intravenous catheters should be premeasured before insertion.

\section{Urinary catheter placement}

A 24-gauge catheter is used to identify and dilate the small, slit-like urethral opening located on the ventral surface of the penis just proximal to the J-shaped curve (Fig. 1). The catheter should be gently inserted between the grooves of the two osseous projections of the os penis. Once the urethral opening has been dilated with the 24-gauge catheter, it becomes easier to insert and thread the urethral catheter that will be secured in place. If resistance is met when passing the urinary catheter, sterile saline should be used to flush the catheter as it is advanced. If resistance is still met, the clinician should proceed with caution, as urethral tear or rupture can result if too much pressure is applied.

Once the catheter is in place, the bladder should be flushed with sterile saline. There may be substantial hematuria (clot formation) or pyuria present, which can obstruct the outflow from the urinary catheter. Tape tabs should be placed on the urinary catheter at the location of the prepuce to suture it to the skin and secure it in place. Sterile tubing (typically intravenous line tubing) is then attached to the urinary catheter and secured with tape and a body wrap to the ferret. A bandage is often applied to the ferrets' torso to prevent the urinary catheter from being pulled out (Fig. 2). A sterile fluid bag is attached to the tubing, creating a closed system for urine collection.

\section{Monitoring after catheter placement}

Urine production should be monitored closely. Urinary output should be at least 1-2 $\mathrm{ml} / \mathrm{kg} / \mathrm{h}$; with diuresis, this may increase substantially. The urinary catheter should be maintained for 1-3 days. Anuria may indicate renal failure. If urine production is insufficient, the line should be disconnected and flushed with sterile saline in case the catheter is kinked, a clot has formed or other mucus or debris is blocking urine outflow from the catheter.

It is recommended to fit ferrets with an Elizabethan collar at the time of catheter placement. A standard collar can be placed on the ferret with a piece of foam pipe insulation between the collar and the shoulders to keep the collar from slipping back along the ferret's neck ${ }^{4}$ (Fig. 3 ).

\section{Alternatives to catheter placement}

When attempts to pass a urinary catheter fail, urine can be removed once via cystocentesis to reduce pressure and allow passage of the urinary catheter. Repeated cystocentesis is not recommended because this carries a risk of urinary bladder rupture and uroabdomen. If normograde passage of a urinary catheter is unsuccessful, consideration should be given to an emergency cystotomy or tube cystostomy ${ }^{5}$. In cases of urethral rupture, an emergency perineal urethrostomy is required, and in some instances, it may be necessary to 'marsupialize' the prostate gland 6 .

\section{Adjunct therapy for adrenal disease}

Leuprolide acetate (Lupron Depot 30 day, TAP Pharmaceutical Products, Inc., Lake Forest, IL), a gonadotropin-releasing hormone analog, is used to decrease androgenic hormone production in ferrets with adrenal disease. Its presumed mechanism in ferrets is to cause a brief rise and then prolonged reduction in levels of luteinizing hormone and folliclestimulating hormone. Leuprolide acetate should be given intramuscularly at $200 \mu \mathrm{g}$

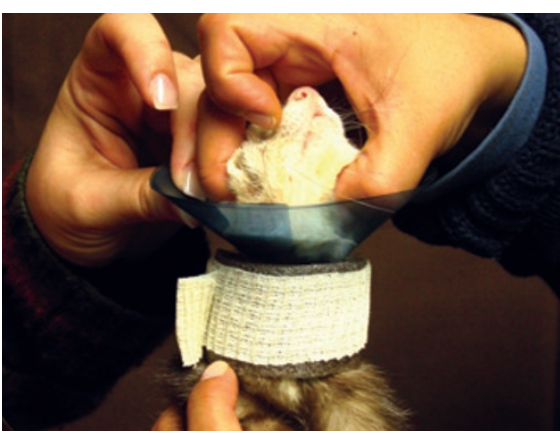

FIGURE 3 | A restraint collar is used to prevent the ferret from removing the urinary catheter. A standard flange restraint collar (made from radiograph film) is placed on a ferret, and a piece of foam pipe insulation is placed behind the collar along the neck to prevent the ferret from pushing the collar back away from his face.

per animal, as it can shrink the prostate within 12-48 hours, which may improve urine flow or the ability to pass a urinary catheter in cases of urethral obstruction associated with adrenal gland disease.

\section{Prognosis}

The prognosis is good for urethral or cystic calculi with aggressive treatment. There is some risk of potential complications, the most pressing being the development of acute renal failure secondary to urethral obstruction. Another serious potential complication is a urethral tear, which would require surgical intervention with a perineal urethrostomy. The prognosis for ferrets with underlying adrenal disease can be complicated; discussion of management of adrenal gland disease in the ferret is beyond the scope of this column.

1. Ball, R.S. Issues to consider for preparing ferrets as research subjects in the laboratory. ILAR J. 47, 348-357 (2006).

2. Pollock, C.G. in Ferrets, Rabbits, and Rodents: Clinical Medicine and Surgery 2nd edn. (eds. Quesenberry K.E. \& Carpenter J.W.) 41-49 (Philadelphia, WB Saunders, 2003).

3. Lennox, A.M. \& Lichtenberger M. A new type of urinary catheter for catheterization of the male ferret. Exotic DVM 10, 5-6 (2008).

4. Brown, C. Restraint collars. Part I: Elizabethan collars and other types of restraint collars. Lab Anim. (NY) 35, 23-25 (2006).

5. Nolte, D.M., Carberry, C.A., Gannon, K.M. \& Boren, F.C. Temporary tube cystostomy as a treatment for urinary obstruction secondary to adrenal disease in four ferrets. J. Am. Anim. Hosp. Assoc. 38, 527-532 (2003).

6. Orcutt, C.J. Treatment of urogenital disease in ferrets. Exotic DVM 3, 31-37 (2001). 